\section{International Scientific Journal Theoretical \& Applied Science}

p-ISSN: 2308-4944 (print) e-ISSN: 2409-0085 (online)

Year: $2016 \quad$ Issue: 1 Volume: 33

Published: $30.01 .2016 \quad \underline{\text { http://T-Science.org }}$

SECTION 7. Mechanics and machine construction.
Mukatay Nemerebayev doctor of technical Sciences, Professor, Taraz innovation and humanities University, Kazakhstan nemerebayev@mail.ru

Murat Musabekovich Bekmuratov Candidate of technical sciences, associate Professor, Taraz state University named after M.Kh. Dulati,

Kazakhstan

Seitzhan Orynbayev

$\mathrm{PhD}$, associate Professor,

Taraz state University named after M.Kh. Dulati,

Kazakhstan

seitzhan_74@mail.ru

Aigerim Nemerebayeva

Master of Laws,

Taraz innovation and humanities University, Kazakhstan

\title{
EFFECT OF SHEAR DEFORMATION ON FREE VIBRATION OF CYLINDRICAL COMPOSITE NETLIKE SHELLS
}

\begin{abstract}
This paper describes the effect of shear deformation on free vibration of composite netlike cylindrical shell.

Using "additional" forces $N_{i i}, N_{i j}$ at critical deviation equilibrium of shell in the linear equation, derived from technical theory, and displacement fields $u, v$, and $w$, defining the relative deformation, derived the limited equation of free vibration of netlike cylindrical shells.

Also based on proven theory of S.A.Ambartsumyan and using displacement fields $u, v$, and $w$ derived the equation for free vibration frequency of netlike cylindrical shell with the effect of shear deformation.

The relative difference $\delta(m, n)$ of the two vibration frequency equations at constant $n=3, n=10$ was plotted against increasing $m$ and it was proven that the difference increases with the effect of shear deformation.

Key words: vibration, deformation, frequency, shell.

Language: English

Citation: Nemerebayev M, Bekmuratov MM, Orynbayev S, Nemerebayeva A (2016) EFFECT OF SHEAR DEFORMATION ON FREE VIBRATION OF CYLINDRICAL COMPOSITE NETLIKE SHELLS. ISJ Theoretical \& Applied Science, 01 (33): 86-90.

Soi: http://s-o-i.org/1.1/TAS-01-33-17 Doi: crossef http://dx.doi.org/10.15863/TAS.2016.01.33.17
\end{abstract}

\section{Introduction.}

One of the problems of used shell theories based on Kirchoff-Love kinematical model is that it does not account for shear deformation. This factor effects determining the vibration frequency and critical static force, thus there is no need to prove that it will have its impact when solving complex dynamic problems. This paper describes the use of technical theory based on Kirchoff-Love kinematical model on composite cylindrical netlike shells.

Nowadays when solving problems of plates and shells taking into account shear deformation, the linear theory of cross-sectional tangent deflections of structures (The Timoshenko kinematic model) and the theory based on parabolic shear stress on crosssection are mostly used $[1,2,3]$.
Analyzing the papers describing the determination the frequency of free vibration using the shear deflection and rotatory inertia of isotropic cylindrical shells Greenberg et al [4] points on a problem of determining the free vibration frequency of anisotropic shells especially the effect of shear deformation on shell with low shear module.

2. The method to account for the effect of shear deformation on free vibration of composite netlike cylindrical shell.

From the technical theory we can derive the following linear equations for netlike cylindrical shell.

$$
\frac{\partial N_{11}}{\partial x}+\frac{\partial N_{21}}{\partial y}+P_{1}+X_{1}=0
$$




$$
\begin{gathered}
\frac{\partial N_{22}}{\partial y}+\frac{\partial N_{12}}{\partial x}+P_{2}+X_{2}=0, \\
\frac{\partial N_{13}}{\partial x}+\frac{\partial N_{23}}{\partial y}-\frac{N_{22}}{R}+P_{3}+X_{3}=0, \\
\frac{\partial M_{H}}{\partial x}+\frac{\partial M_{21}}{\partial y}-N_{13}+\Phi_{2}+F_{2}=0, \\
\frac{\partial M_{22}}{\partial y}+\frac{\partial M_{12}}{\partial x}-N_{23}+\varphi_{1}+F_{1}=0,
\end{gathered}
$$

here: $N_{i j}$, are the "additional" forces at critical deviation equilibrium of shell, and under the technical theory will take the following values.

$$
\begin{aligned}
& N_{11}=C_{11} \varepsilon_{1}+C_{12} \varepsilon_{2} \text {, } \\
& N_{22}=C_{22} \varepsilon_{2}+C_{12} \varepsilon_{1} \text {, } \\
& N_{12}=N_{21}=C_{66} \varepsilon_{12} \text {, } \\
& N_{13}=C_{13}\left(\theta_{1}+\gamma_{2}\right) \\
& N_{23}=C_{23}\left(\theta_{2}+\gamma_{y}\right) \text {, } \\
& M_{11}=D_{11} x_{1}+D_{12} x_{2} \text {, } \\
& C_{11}=\frac{\partial^{2} u}{\partial x^{2}}+C_{66} \frac{\partial^{2} u}{\partial y^{2}}+\left(C_{12}+C_{66}\right) \frac{\partial^{2} v}{\partial x \partial y}+\frac{c_{12}}{R} \frac{\partial \omega}{\partial x}=\frac{2 \rho h \delta}{a}\left(\frac{\partial^{2} u}{\partial t}+\frac{h^{2} \delta^{2}}{3 a^{2} R} \frac{\partial^{2} \gamma_{x}}{\partial t^{2}}\right) ; \\
& \left(C_{12}+C_{66}\right) \frac{\partial^{2} u}{\partial x \partial y}+C_{22} \frac{\partial^{2} v}{\partial y^{2}}+C_{66} \frac{\partial^{2} v}{\partial x^{2}}+\frac{C_{22}}{R} \frac{\partial w}{\partial y}=\frac{2 \rho h \delta}{a}\left(\frac{\partial^{2} u}{\partial t}+\frac{h^{2} \delta^{2}}{3 a^{2} R} \frac{\partial^{2} \gamma_{x}}{\partial t^{2}}\right) \\
& C_{13} \frac{\partial^{2} \omega}{\partial x^{2}}+C_{23} \frac{\partial^{2} \omega}{\partial y^{2}}-\frac{C_{22}}{R} \omega-\frac{C_{12}}{R} \frac{\partial u}{\partial x}-\frac{C_{22}}{R} \frac{\partial \vartheta}{\partial y}+C_{13} \frac{\partial \gamma x}{\partial x}+C_{23} \frac{\partial \gamma_{y}}{\partial y}=\frac{2 \rho h \delta}{a} \frac{\partial^{2} \omega}{\partial t^{2}} \text {; } \\
& D_{11} \frac{\partial^{2} \gamma_{x}}{\partial x^{2}}+D_{66} \frac{\partial^{2} \gamma_{x}}{\partial y^{2}}-C_{13} \gamma_{x}+\left(D_{12}+D_{66}\right) \frac{\partial^{2} \gamma_{y}}{\partial x \partial y}-C_{13} \frac{\partial \omega}{\partial x}=\frac{2 \rho h^{3} \delta^{3}}{3 a^{3}}\left(\frac{\partial^{2} \gamma_{x}}{\partial t^{2}}+\frac{1}{R} \frac{\partial^{2} u}{\partial t^{2}}\right) \\
& D_{22} \frac{\partial^{2} \gamma_{y}}{\partial y^{2}}+D_{66} \frac{\partial^{2} \gamma_{y}}{\partial x^{2}}-C_{23} \gamma_{y}\left(D_{12}+D_{66}\right) \frac{\partial^{2} \gamma_{x}}{\partial x \partial y}-C_{33} \frac{\partial \omega}{\partial y}=\frac{2 \rho h^{3} \delta^{3}}{3 a^{3}}\left(\frac{\partial^{2} \gamma_{y}}{\partial t^{2}}+\frac{1}{R} \frac{\partial^{2} \vartheta}{\partial t^{2}}\right)
\end{aligned}
$$

Assuming that the shell is in free connection with two sides, and at $x=0$ values it is satisfied with finite conditions $N=0 \quad \vartheta=0 \quad \omega=0$

$M_{x}=0$, and putting the unknowns $u, \vartheta, \omega$ and $\gamma_{x}, \gamma_{y}$ into Fourier series we get:

$$
\begin{array}{r}
U(x, y, t)=\sum_{m=1}^{\wp} \sum_{n=1}^{\wp}\left[U_{m n}(t) \cos \beta_{n} y+U_{m n}^{\prime}(t) \sin \beta_{n} y\right] \cos \alpha_{m} x \\
\vartheta(x, y, t)=\sum_{m=1}^{\wp} \sum_{n=1}^{\wp}\left[V_{m n}(t) \sin \beta_{n} y-V_{m n}^{\prime}(t) \cos \beta_{n} y\right] \sin \alpha_{m} x \\
\omega(x, y, t)=\sum_{m=1}^{\wp} \sum_{n=1}^{\wp}\left[W_{m n}(t) \cos \beta_{n} y-W_{m n}^{\prime}(t) \sin \beta_{n} y\right] \sin \alpha_{m} x
\end{array}
$$




\begin{tabular}{l|lrl|l|ll} 
& ISRA (India) & $=\mathbf{1 . 3 4 4}$ & SIS (USA) & $=\mathbf{0 . 9 1 2}$ & ICV (Poland) & $=\mathbf{6 . 6 3 0}$ \\
Impact Factor: & ISI (Dubai, UAE) $=\mathbf{0 . 8 2 9}$ & PUHU (Russia) $=\mathbf{0 . 1 7 9}$ & PIF (India) & $=\mathbf{1 . 9 4 0}$ \\
& GIF (Australia) & $\mathbf{0 . 5 6 4}$ & ESJI (KZ) & $=\mathbf{1 . 0 4 2}$ & & \\
& JIF & $=\mathbf{1 . 5 0 0}$ & SJIF (Morocco) $=\mathbf{2 . 0 3 1}$ & &
\end{tabular}

$$
\begin{aligned}
& \gamma_{x}(x, y, t)=\sum_{m=1}^{\wp} \sum_{n=1}^{\wp}\left[x_{m n}(t) \cos \beta_{n} y+x_{m n}^{\prime}(t) \sin \beta_{n} y\right] \cos \alpha_{m} x \\
& \gamma_{y}(x, y, t)=\sum_{m=1}^{\wp} \sum_{n=1}^{\wp}\left[Y_{m n}(t) \sin \beta_{n} y-Y_{m n}^{\prime}(t) \cos \beta_{n} y\right] \sin \alpha_{m} x .
\end{aligned}
$$

here $\alpha_{m}=\frac{\pi m}{L}, \beta_{n}=\frac{n}{R}$.

Putting equation (6) into equation (5) and by using two similar $U_{m n}, V_{m n}, W_{m n}, X_{m n}, Y_{m n}$ and $U_{m n}^{\prime}, V_{m n}^{\prime}, W_{m n}^{\prime}, X_{m n}^{\prime}, Y_{m n}^{\prime}$ unknowns we get the series of equations, we write the first five differential equations in matrix form.

$$
M \frac{d^{2} f_{m n}}{d t^{2}}+G_{m n} f_{m n}=0
$$

Here $f_{m n}=\left\{U_{m n}, V_{m n}, W_{m n}, X_{m n}, Y_{m n}\right\}$ and $\mathrm{G}_{\mathrm{mn}}$, $\tilde{M}$ are next symmetric matrixes not equal to zero:

$$
\begin{gathered}
g_{m n}^{11}=\alpha_{m}^{2} C_{n}+\beta_{n}^{2} C_{66} ; \\
g_{m n}^{2}=-\alpha_{m} \beta_{n}\left(C_{12}+C_{66}\right) \\
g_{m n}^{13}=-\frac{C_{12}}{R} d m ; \\
g_{m n}^{22}=\alpha_{m}^{2} C_{66}+\beta_{n}^{2} C_{22} ; \\
g_{m n}^{23}=\frac{C_{22}}{R} \beta_{n} ;
\end{gathered}
$$

$$
\begin{gathered}
g_{m n}^{33}=\frac{C_{22}}{R^{2} m}+C_{13} \alpha_{m}^{2}+C_{23} \beta_{n}^{2} ; \\
g_{m n}^{34}=C_{13} \alpha_{m} ; \\
g_{m n}^{35}=-C_{23} \beta_{n} ; \\
g_{m n}^{44}=\alpha_{m}^{2} D_{11}+\beta_{n}^{2} D_{66}+C_{13} ; \\
g_{m n}^{45}=-\alpha_{m} \beta_{n}\left(D_{12}+D_{66}\right) ; \\
m_{11}=m_{22}=m_{33}=\frac{2 \rho h \delta}{a} ; \\
m_{44}=m_{55}=\frac{2 \rho h^{3} \delta^{3}}{3 a^{3}} ; \\
m_{14}=m_{25}=\frac{2 \rho h^{3} \delta^{3}}{3 a^{3} R} .
\end{gathered}
$$

Analizing the effect of shear deformation on vibration frequency at bending vibration conditions in literature, and using equation (5) we get the known equation

$$
\frac{d^{2} W_{m n}}{d t^{2}}+\omega_{m n}^{2} W_{m n}=0
$$

Here $\omega^{2}$ value as per [5] determined as follows:

$$
\begin{gathered}
\omega_{m n}^{2}=\frac{a}{2 \rho h \delta}\left[\frac{\alpha_{m}^{4} C_{66}\left(C_{11} C_{22}-C_{12}^{2}\right)}{\Delta_{m n}}+\frac{K_{m n}}{B_{m n}}\right] ; \\
\left.\Delta_{m n}=\left.R^{2}\right|_{\alpha_{m}}{ }^{4} C_{11} C_{66}+\beta_{n}{ }^{4} C_{22} C_{66}+\alpha_{m}{ }^{2} \beta_{n}{ }^{2}\left(C_{11} C_{22}-C^{2}{ }_{12}-2 C_{12} C_{66}\right)\right] \\
K_{m n}=\alpha_{m}{ }^{6} C_{13} D_{11} D_{66}+\alpha_{m}^{4} \beta_{n}{ }_{n}\left[C_{13}\left(D_{11} D_{22}-D^{2}{ }_{12}-2 D_{12} D_{66}\right)+C_{23} D_{11} D_{66}\right]+ \\
\alpha_{m}^{2} \beta_{n}^{4}\left[C_{13} D_{22} D_{66}+C_{23}\left(D_{11} D_{22}-D_{12}{ }^{2}-2 D_{12} D_{66}\right)\right]+\beta_{n}{ }^{6} C_{23} D_{22} D_{66}+C_{13} C_{23} \times \\
\left.\left.\alpha_{m}{ }^{4} D_{11}+\beta_{n}{ }^{4} D_{22}+2 \alpha_{m}{ }^{2} \beta_{n}{ }^{2}{ }_{n}\left(D_{12}+2 D_{66}\right)\right]\right] \\
B_{m n}=\alpha_{m}{ }^{4} D_{11} D_{66}+\alpha^{2}{ }_{m} \beta_{n}{ }^{2}\left(D_{11} D_{22}-D^{2}{ }_{12}-2 D_{12} D_{66}\right)+ \\
\beta_{4}{ }^{4} D_{22} D_{66}+\alpha^{2}{ }_{m}\left(C_{13} D_{66}+C_{23} D_{11}\right)+\beta_{n}{ }^{2}\left(C_{13} D_{22}+C_{23} D_{66}\right)+C_{13} C_{23}
\end{gathered}
$$

$C_{i j}$ and $D_{i j}$ are hardness of shell [6].
If $C_{13} \rightarrow \infty, C_{23} \rightarrow \infty$ then from equation (8) as per [4] we get below vibration equation based on technical theory: 


\begin{tabular}{l|lrl|l|ll} 
& ISRA (India) & $=\mathbf{1 . 3 4 4}$ & SIS (USA) & $=\mathbf{0 . 9 1 2}$ & ICV (Poland) & $=\mathbf{6 . 6 3 0}$ \\
Impact Factor: & ISI (Dubai, UAE) $=\mathbf{0 . 8 2 9}$ & PUHL (Russia) & $=\mathbf{0 . 1 7 9}$ & PIF (India) & $=\mathbf{1 . 9 4 0}$ \\
& GIF (Australia) & $\mathbf{0 . 5 6 4}$ & ESJI (KZ) & $=\mathbf{1 . 0 4 2}$ & & \\
& JIF & $\mathbf{1 . 5 0 0}$ & SJIF (Morocco) & $=\mathbf{2 . 0 3 1}$ & & \\
\hline
\end{tabular}

$$
\omega_{m n}^{2}=\frac{2}{2 \rho h \delta}\left[\alpha_{m}^{4} D_{11}+2 \alpha_{m}^{2} \beta_{n}^{2}\left(D_{12}+2 D_{66}\right)+\beta_{n}^{4} D_{22}+\frac{\alpha_{m}^{4} C_{66}\left(C_{11} C_{22}-C_{12}^{2}\right)}{\Delta_{m n}}\right] .
$$

If we use proved equations of S.A. Ambartsumyan we get as a base of analysis the next values of shear stresses

$$
\begin{aligned}
& \sigma_{13}=\frac{1}{2}\left(\frac{h^{2} \delta^{2}}{a^{2}}-z^{2}\right) \varphi(x, y, t) ; \\
& \sigma_{23}=\frac{1}{2}\left(\frac{h^{2} \delta^{2}}{a^{2}}-z^{2}\right) \psi(x, y, t) . \\
& u_{1}=u+z\left[-\frac{\partial \omega}{\partial \mathrm{X}}+a_{55} \varphi\left(\frac{h^{2} \delta^{2}}{a^{2} 2}-\frac{z^{2}}{6}\right)\right] ; u_{2}=v+z\left[-\frac{\partial w}{\partial y}+a_{44} \psi\left(\frac{h^{2} \delta^{2}}{2 a^{2}}-\frac{z^{2}}{6}\right)\right] \\
& \varepsilon_{11}=\frac{\partial u}{\partial x}+z\left[-\frac{\partial^{2} w}{\partial x^{2}}+a_{55} \frac{\partial \varphi}{\partial x}\left(\frac{h^{2} \delta^{2}}{2 a^{2}}-\frac{z^{2}}{6}\right)\right] \\
& \varepsilon_{22}=\frac{\partial v}{\partial y}+\frac{w}{R}+z\left[-\frac{\partial^{2} w}{\partial y^{2}}+a_{44} \frac{\partial \psi}{\partial y}\left(\frac{h^{2} \delta^{2}}{2 a^{2}}-\frac{z^{2}}{6}\right)\right] \\
& \varepsilon_{12}=\frac{\partial u}{\partial y}+\frac{\partial v}{\partial x}+z\left[-2 \frac{\partial^{2} w}{\partial x \partial y}+\left(a_{55} \frac{\partial \varphi}{\partial y}+a_{44} \frac{\partial \psi}{\partial x}\right)\left(\frac{(h \delta)^{2}}{2 a^{2}}-\frac{z^{2}}{6}\right)\right] \text {. }
\end{aligned}
$$

Here $a_{55}=\frac{1}{G_{13}}, a_{44}=\frac{1}{G_{23}}, G_{i 3}$ - shear module.

The intersecting forces and momentums values are defined as follows:

$$
\begin{gathered}
N_{13}=\frac{2(h b)^{3}}{3 a^{3}} \varphi ; N_{23}=\frac{2(h b)^{3}}{3 a^{3}} \varphi \\
M_{11}=D_{11} \frac{\partial}{\partial x}\left(-\frac{\partial w}{\partial x}+a_{55} \frac{2(h b)^{2}}{5 a^{2}} \varphi\right)+D_{12} \frac{\partial}{\partial y}\left(-\frac{\partial w}{\partial y}+a_{44} \frac{2(4 b)^{2}}{5 a^{2}} \varphi\right) \\
M_{22}=D_{12} \frac{\partial}{\partial x}\left(-\frac{\partial w}{\partial x}+a_{55} \frac{2(h b)^{2}}{5 a^{2}} \varphi\right)+D_{22} \frac{\partial}{\partial y}\left(-\frac{\partial w}{\partial y}+a_{44} \frac{2(h b)^{2}}{5 a^{2}} \varphi\right) \\
M_{12}=M_{21}=D_{66}\left(\frac{\partial}{\partial x}\left(-\frac{\partial w}{\partial y}+a_{44} \frac{2(4 b)^{2}}{5 a^{2}} \varphi\right)+\frac{\partial}{\partial y}\left(-\frac{\partial w}{\partial x}+a_{55} \frac{2(4 b)^{2}}{5 a^{2}} \varphi\right)\right)
\end{gathered}
$$

The movement equation of the shell is described with unknowns $u, v, w, \varphi$, thus putting the values of intersecting forces and momentums from equation (13) to equation (1), and also considering the values of

$$
\begin{aligned}
& X_{x}=\frac{\partial w}{\partial x}+a_{55} \frac{2(4 b)^{2}}{5 a^{2}} \varphi \\
& \gamma_{y}=-\frac{\partial w}{\partial y}+a_{44} \frac{2(4 b)^{2}}{5 a^{2}} \varphi \text { and } \\
& C_{13}=K^{1} \frac{2 h b}{a} G_{13}
\end{aligned}
$$

$$
\begin{aligned}
& G_{23}=K^{11} \frac{2 h b}{a} G_{23} \\
& K^{1}=K^{11}=5 / 6 \text { we get the equation (9). }
\end{aligned}
$$

This means that the equation taken as per proven theory of S.A. Ambartsumyan is derived as per above equation (9) of netlike shell. If comparing the $\left(\omega_{m n}\right)$ values of above equation (9) with equation (10), then the relative difference will be the difference of $\delta_{(m n)}=\frac{\omega_{m n}^{(1)}}{\omega_{m n}^{(10)}}-1$ values. 


\begin{tabular}{l|lrl|l|ll} 
& ISRA (India) & $=\mathbf{1 . 3 4 4}$ & SIS (USA) & $=\mathbf{0 . 9 1 2}$ & ICV (Poland) & $=\mathbf{6 . 6 3 0}$ \\
Impact Factor: & ISI (Dubai, UAE) $=\mathbf{0 . 8 2 9}$ & PUHU (Russia) $=\mathbf{0 . 1 7 9}$ & PIF (India) & $=\mathbf{1 . 9 4 0}$ \\
& GIF (Australia) & $\mathbf{0 . 5 6 4}$ & ESJI (KZ) & $=\mathbf{1 . 0 4 2}$ & & \\
& JIF & $=1.500$ & SJIF (Morocco) & $=\mathbf{2 . 0 3 1}$ & &
\end{tabular}

When $\mathrm{R}=1, \mathrm{~L} / \mathrm{R}=2$ and because $\mathrm{m}$ and $\mathrm{L}$ values are in combination of $\frac{\pi m R}{L}$ we can analyze the change of $\mathrm{m}$ while $1 / \mathrm{L}$ is constant, or vice versa.
Putting the equation into program we can see that at $\mathrm{n}=3, \mathrm{n}=10$ and $\mathrm{G}_{12} / \mathrm{G}_{13}=1, \delta$ increases.
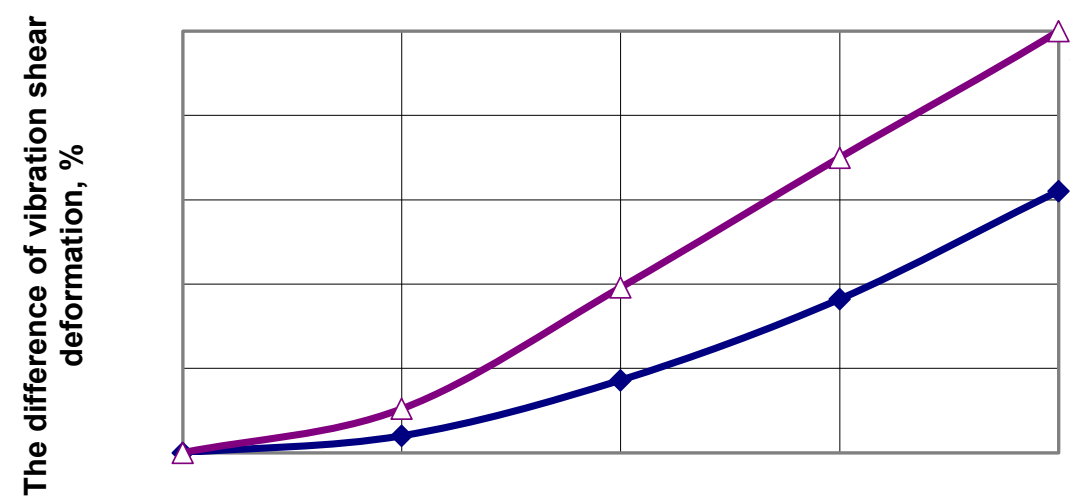

The form of linear vibration, $m$

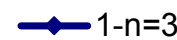

$\Delta-2-n=10$

Figure 1 -The graph of changing the difference of vibration using shear deformation, where $n=3, n=10$, from $m$.

\section{References:}

1. Ambartsumian SA (1974) The general theory of anisotropic shells. - Moscow: Nauka, 1974, $448 \mathrm{p}$.

2. Junisbekov TM, Kestelman VN, Malinin N (2002) Stress Relaxation in viscolastic Materials //Science Publishers, Inc., Enfield, NM, USA 2002.- 458 p.

3. Greenberg IB, Stavsky Y (1980) Vibrations of axially compressed laminated orthotropic cylindrical shells, including transverse shear deformation.// Acta Mech, 1980. Vol. 37, №12, pp. 13-28.

4. Rao SYVK (1983) Vibrations of layered shells with transverse shear and rotatory inertia effects // I. Sound a. Vibration, 1983, vol 86, №1, pp. 147-150.

5. Nemerebaev M (2007) Cylinder tarizdi composite materialdary zhasalgan torus kozdi kabyrshyқty erkin terbeliske esepteu // Karagandy tehnikalyk universitettin gylymi enbekter zhinagy, Karagandy, №2, 2007, pp. 124-132.

6. Nemerebaev M (2006) Cylinder tarizdi composite materialdary zhasalgan torus kozdi kabyrshyktyn syzyksyz tendeui // MTPU, Taraz, №2, 2006, pp. 249-258.

7. Nurimbetov A, Bekbayev A, Orynbayev S, Dzhamanbayev M, Keikimanova M (2015) Naturally Twisted Layered Anisotropic Rod Made of Reinforced Materials Research. Applied Mechanics and Materials, 736, pp.3038.

8. Nurimbetov A, Bekbayev A, Orynbayev S, Baibutanov B, Tumanov I, Keikimanova M (2015) Optimization of windmill's layered composite Blades to reduce Aerodynamic Noise and Use in Construction of "Green" Cities // Procedia Engineering. Vol. 117, 2015. pp.273287.

9. Krenk S (1982) The Torsion-Extension Coupling in Pretwisted Elastic Beams International Journal of Solids and Structures, 19 (1), 1982. pp. 67-72.

10. Hodqes DH (1980) Torsion of Pretwisted Beams Due to Axial Loading Journal of Applied Mechanics, 42 (7), 1980. pp. 393-397. 